Cómo citar este artículo: Piratoba, R P. \& Rojas, C. E. (2014). Cambios en las concepciones iniciales e inducidas sobre la naturaleza de las matemáticas y su didáctica, en estudiantes de un programa de licenciatura en matemáticas y estadística. Rev.investig.desarro.innov, 5(1), 32-45

\title{
CAMBIOS EN LAS CONCEPCIONES INICIALES E INDUCIDAS SOBRE LA NATURALEZA DE LAS MATEMÁTICAS Y SU DIDÁCTICA, EN ESTUDIANTES DE UN PROGRAMA DE LICENCIATURA EN MATEMÁTICAS Y ESTADÍSTICA
}

\section{CHANGES ON THE INITIAL CONCEPTIONS AND INDUCTION ON THE NATURE OF MATHEMATICS AND ITS DIDACTICS OF AN UNDERGRADUATE STUDENTS PROGRAM IN MATHEMATICS AND STATISTICS}

\section{Ruth Paola Piratoba Gil 1 Clara Emilse Rojas Morales ${ }^{2}$}

Recibido: febrero 22 de 2014

\section{Resumen}

El presente documento muestra algunos resultados de una investigación cuyo objetivo fue el de indagar las concepciones que tienen los estudiantes en un programa de formación inicial para profesores de matemáticas, sobre la naturaleza de las matemáticas, su forma de aprender y enseñar. Se describen en forma general los referentes teóricos del proyecto, así como las categorías de análisis establecidas para el diseño y validación de un instrumento que permitió identificar las concepciones de estudiantes de la Licenciatura en Matemáticas y Estadística, de la Universidad Pedagógica y Tecnológica de Colombia Facultad Duitama. Se resaltan algunas técnicas estadísticas usadas para el análisis de la información recopilada, concluyendo que los estudiantes de primer semestre conservan una visión estática del cómo se enseñan y evalúan las matemáticas, mientras que los de terminación académica, han modificado sus concepciones, al considerar la matemática como una construcción humana y social en constante dinamismo, aunque con ligeros visos de rigidez en cuanto a su evaluación.

Palabras clave: naturaleza de las matemáticas, concepciones, técnicas de análisis, didáctica de las matemáticas.

\section{Abstract}

This paper shows some results of a study whose objective was to investigate the conceptions that students have about a basic training program for teachers of mathematics, about the nature of mathematics, and the way they learn and teach. The theoretical framework of the project is described in general terms as well as the analytical categories established for the design and validation of an instrument that identified the conceptions of students of the Licenciatura of Mathematics and Statistics, at Pedagogical and Technological University of Colombia, Duitama Branch. Some statistics techniques used to analyze the collected information are highlighted, concluding that freshmen retain a static view of how to teach and assess maths, while the students from the last semesters have modified their conceptions, considering mathematics as a human and social construction in constant dynamism, although with slight overtones of rigidity in their evaluation.

Key words: qualitative research, concepts, analysis techniques, questionnaire, mathematics nature and mathematics didactic.

\footnotetext{
1 Licenciada en Matemáticas y Estadística. Docente Colegio Seminario Diocesano de Duitama. E-mail: rutkis3120@hotmail.com 2 Magister en docencia matemática. Docente Universidad Pedagógica y Tecnológica de Colombia. E-mail: claritain@yahoo.es
} 


\section{Introducción}

En el marco de políticas educativas nacionales sobre formación de profesores, se establecen criterios para que las Instituciones de Educación Superior ofrezcan una formación profesional de la más alta calidad e idoneidad.

En este contexto, la Universidad Pedagógica y Tecnológica de Colombia, UPTC, ofrece desde hace 34 años, la Licenciatura en Matemáticas y Estadística, LME, en la Facultad Seccional Duitama. A partir de 2010 la LME tiene una duración de 10 semestres académicos, ya que antes de ese año era un programa de modalidad nocturna, con duración de 12 semestres. La licenciatura tiene el propósito de formar educadores matemáticos autónomos, reflexivos e innovadores, responsables en la construcción de proyectos de vida, capaces de participar activamente en el desarrollo integral de los educandos así como el mejoramiento de la calidad de la Educación y la transformación de la realidad individual y social del mundo actual.

El proyecto curricular de la LME considera que el conocimiento profesional del profesor de matemáticas se conforma a través de la explicitación de las creencias y concepciones iniciales sobre las matemáticas, la enseñanza y el aprendizaje, el cuestionamiento y confrontación de estas concepciones con las teorías pedagógicas y didácticas que se estudian a lo largo del programa, requiriendo una implicación personal en la práctica y existencia de coherencia con el modelo didáctico subyacente a la formación (Azcárate, 1998; Blanco, 1998).

El proyecto curricular contempla dentro de su formación profesional, lograr una formación Disciplinar en el área de Matemáticas y Estadística en la cual se profundice tanto en el conocimiento Matemático y Estadístico como en la naturaleza de las matemáticas disciplinares y escolares; además busca lograr una formación Pedagógica y Didáctica, en la cual se articulen saberes teóricos y prácticos y, mediante la reflexión y sistematización de experiencias educativas, generar conocimientos pedagógicos y didácticos acordes a las actuales expectativas sociales y culturales de la Educación Matemática.

A los profesores formadores de futuros profesores les interesa saber en qué forma está incidiendo el Proyecto curricular de la LME, para el desarrollo de su conocimiento profesional y qué competencias se están fomentando para desempeñarse con idoneidad en su vida profesional. Pero esta preocupación no es exclusiva de la licenciatura, hoy en el campo de la Educación Matemática y específicamente en la línea de investigación de formación y desarrollo profesional de los profesores de matemáticas, se hallan numerosas investigaciones que han identificado que las creencias y concepciones que tienen los profesores sobre las matemáticas, determina su elección de las actividades, del ambiente de aprendizaje generado, así como el discurso de la clase (García, Azcarate y Moreno, 2006; Blanco, 1998; Zapata, Blanco y Contreras, 2008; Flores, 1998; Llinares, 1991). Es decir, la concepción que tenga el docente sobre la naturaleza de las matemáticas y la actividad matemática incide directamente en las concepciones sobre la forma de enseñar, de aprender y de evaluar las matemáticas (Gámez, Moreno y Gil, 2003).

La comunidad de investigadores sobre la formación inicial y continuada de profesores está de acuerdo en señalar la importancia del estudio de las concepciones de los profesores en ejercicio y en formación, para promover reflexión y mejoras en los procesos de enseñanza y aprendizaje de las matemáticas (Azcárate y Cardeñoso, 1998). Otros resaltan la importancia de estudiar las concepciones y creencias de los profesores de matemáticas ya que encuentran una gran relación de éstas con la experiencia en el aula de clase, interfiriendo en ellas sus conocimientos, juicios, expectativas y convicciones sobre la manera en la que transmiten los conocimientos a los estudiantes (Caballero, Blanco y Guerrero, 2007; 
D’Amore y Fandiño, 2004). Algunos autores han hablado sobre la reflexión crítica del docente sobre su propia práctica en diversos contextos significativos, aspecto importante ya que se ha evidenciado como, con el tiempo, este aspecto genera un cambio en las concepciones y en las creencias con respecto a la formación profesional que recibieron (Godino, Batanero y Font, 2004; Jiménez, 2009; García, 2000; Dodera, Burroni, Lázaro y Piacentini. 2008). Probablemente esta idea es una de las causas por las que se ha intensificado la investigación en la formación de los profesores, cuyos resultados están sugiriendo cambios profundos en los programas de formación.

De acuerdo con Azcárate (1998), los estudiantes para profesores poseen ciertas concepciones relativas a los procesos de enseñanza y aprendizaje de la Matemática; por ello, el trabajo desarrollado durante el período de formación ha de partir de la explicitación, cuestionamiento y, si es posible, de la movilización de esas ideas. La formación inicial es considerada como la primera fase del proceso de desarrollo profesional y en ella se ponen las bases para la elaboración de un conocimiento práctico profesional, que refleje un modelo didáctico personal cada vez más explícito.

La investigación pretende identificar cuáles son las concepciones iniciales con que ingresan los estudiantes al programa de LME, estudiando si hay cambios o modificaciones de tales concepciones por la incidencia de los componentes disciplinar, investigativo, didáctico y pedagógico del plan de estudios. Es de interés para el grupo de investigación en Educación Matemática y Estadística, EDUMAES, el determinar algunos factores que favorecen u obstaculizan la evolución de las concepciones sobre la naturaleza de las matemáticas, su enseñanza y aprendizaje, cuando los estudiantes ingresan a la licenciatura (concepciones iniciales o espontáneas) y cuando han cursado el $80 \%$ del proyecto curricular del programa (concepciones inducidas pero no controladas por el currículo de formación). Lo anterior llevará a la búsqueda de estrategias y acciones que propicien el mejoramiento del proyecto curricular para lograr los objetivos y el perfil profesional deseado.

En la investigación aquí reportada se desarrollaron dos fases, una relacionada con la construcción del marco teórico que permitió ubicar dentro de la literatura de la Educación Matemática, los términos creencia, concepción, naturaleza de las matemáticas y su evolución histórica, así como las implicaciones didácticas, representadas en el cómo enseñar, aprender y evaluar las matemáticas; ésta primera fase se sintetizó en el artículo de Cerón, Mesa y Rojas (2012). En su segunda etapa, se recopiló y analizó información mediante el diseño y validación de un cuestionario que permitió identificar dichas concepciones en los estudiantes de la LME de la UPTC, acerca de lo que consideran, es el conocimiento matemático, su forma de enseñarlo y aprenderlo, siendo este el propósito del presente reporte.

A continuación se describen los resultados de aplicar un cuestionario para identificar las concepciones sobre la naturaleza de las matemáticas en estudiantes de primer semestre y los de terminación académica (décimo y décimo primer semestre) del programa de LME. Fue posible establecer el impacto positivo del proceso formativo, a través del cambio observado en las concepciones y creencias de los estudiantes, lo cual resalta la importancia que tiene la didáctica de las matemáticas en el aula para su futuro ejercicio docente.

\section{Materiales y métodos}

\subsection{Referente teórico}

Dentro del marco de las investigaciones sobre concepciones y creencias de los futuros docentes, el grupo de investigación EDUMAES opta por la postura de Ponte (1994) citado por (Cerón, Mesa y Rojas, 2012), quien establece que: “Las creencias y 
concepciones tienen una función cognitiva, es decir, forman parte del conocimiento, bien sea la base o la organización de este. Las creencias están ligadas a las actitudes, las expectativas derivadas de la experiencia o fantasía, mientras que las concepciones son los marcos conceptuales con presupuestos teóricos con naturaleza esencialmente cognitiva que condicionan la forma en que se afrontan situaciones. De ahí que es de interés, el estudiar las concepciones en términos que ellas juegan un papel esencial en el pensamiento y la acción del profesor, que redunda en el desarrollo de currículo, procesos de enseñar e investigar en matemáticas".

A la luz de la postura anterior, se establecieron dos macro-concepciones desde una perspectiva histórica sobre la naturaleza de las matemáticas, considerando a su vez las características entre las corrientes filosóficas, estas son: Visión estática (platonismo, logicismo y formalismo) y Visión dinámica (aristotelismo, empirismo, intuicionismo, cuasi-empirismo y constructivismo), en donde se reconocen cuatro factores, caracterizados de la siguiente manera:

Conocimiento matemático: se intenta caracterizar mediante indicadores, qué son las matemáticas desde la visión estática (matemática rígida, terminada y formal) y dinámica (matemática en construcción y falible).

Enseñanza: en esta se ubican las sub-categorías: Concepción acerca de la enseñanza para la visión estática y la visión dinámica, el rol del profesor, la metodología y la relación profesor-estudiante; los indicadores que se establecen, muestran un perfil de lo que se puede reconocer dentro de las visiones para éste proceso.

Aprendizaje: se ubican las sub-categorías: concepción sobre el aprendizaje, el rol del estudiante, la relación estudiante-profesor y se suma un aspecto que a la hora de enseñar, a veces no se tiene en cuenta, como es la motivación frente al aprendizaje en los estudiantes; al igual que en las otras categorías los indicadores ayudan a estudiar la posible tendencia (visión estática y visión dinámica).

Evaluación: se definen como subcategorías los instrumentos que se utilizan y la concepción que sobre ésta hay desde la visión estática y visión dinámica, los indicadores con respecto a esta categoría están dirigidos a describir precisamente el uso de los mencionados instrumentos (Cerón et al., 2012).

Teniendo claras las categorías desde la postura de las concepciones, se llevó a cabo el estudio estadístico. En este sentido, se diseñó un cuestionario teniendo en cuenta las categorías ya establecidas las cuales son el resultado de la fundamentación teórica realizada en la primera parte de la investigación del grupo EDUMAES denominada: Las concepciones sobre la naturaleza de las matemáticas y sus implicaciones didácticas (Cerón et al., 2012).

El análisis de los datos se llevó a cabo en dos etapas: la primera consistió en un análisis descriptivo de cada una de las preguntas, para observar el comportamiento de los estudiantes que comienzan y finalizan sus estudios en el programa; en la segunda etapa se efectúa un análisis multivariado de los datos, empleando el software estadístico R. Para el análisis multivariado, que trata de datos asociados a conjuntos de medidas sobre un número de individuos $\mathrm{u}$ objetos, se utilizaron las siguientes técnicas (Peña, 2000; Díaz, 2002):

Componentes principales: es una técnica que consiste en generar nuevas variables que expresen información contenida en un conjunto de datos: Reduce la dimensión del espacio donde están inscritos los datos, eliminar las variables (si es posible) que aporten poco al estudio del problema, facilitar la interpretación de la información contenida en los datos, determina 
factores que tengan mayor variabilidad en los datos, las variables pueden ser independientes bajo el supuesto de normalidad y no correlación.

Análisis discriminante: consiste en la separación o discriminación de grupos: En este método se obtiene una función que separa varios grupos definidos, esta combinación es lineal, minimiza los errores de clasificación, el análisis de discriminación es para más de dos grupos, hay unas reglas de discriminación:

Clasificación vía máxima verosimilitud: localiza el caso caracterizado por $\mathrm{X}$ en alguna de dos poblaciones, consiste en ubicarlo en la población para la cual $X$ maximiza la verosimilitud o probabilidad. Cuando los datos tienen covarianzas iguales las variables independientes se asumen distribuidos normalmente y la variable respuesta se asume fija, la cual toma los valores cero o uno según la ubicación del objeto en alguno de los dos grupos, y busca también un procedimiento para asignar o clasificar casos o grupos.

Regla de discriminación bayesiana: se basa en el teorema de Bayes, y puede haber una clasificación incorrecta.

Regla de discriminación para varios grupos: se considera el caso de muestras obtenidas a partir de K grupos independientes. Se desarrollan reglas de discriminación para el caso de varias poblaciones que tiene matrices de covarianzas igual o distinta, respectivamente.

Análisis de correlación canónico: busca establecer la relación entre un conjunto de variables predictoras y un conjunto de variables respuesta, el objetivo es encontrar el par de combinaciones lineales, una en cada conjunto, que tengan la correlación más alta entre ellas para determinar si existe algún grado de asociación entre los dos conjuntos de variables; la estrategia consiste en encontrar la correlación entre pares de variables, cada una de las cuales es una combinación lineal de las variables de los respectivos conjuntos.

\subsection{Metodología y muestra}

Se realizó un estudio cualitativo descriptivo tipo encuesta, que conllevó a dos acciones: la primera consistió en el estudio de referentes teóricos desde la parte estadística y educación matemática, que permitió establecer un panorama para el análisis de variables categóricas y cuantitativas, construyendo con claridad el marco teórico desde la estadística para el análisis de los datos recopilados. En la presente investigación las variables son de tipo categóricas y cuantitativas, Ilamadas no-métricas y métricas, la primera definida como "una etiqueta la cual se asigna a los objetos con propósito de clasificarlos, pero no poseen el significado numérico usual, aparente de la relación de igualdad; por tanto tienen naturaleza no-métrica. Las actitudes y las opiniones sobre determinado tema son algunas variables de este tipo"(Díaz, 2002).

La segunda acción se fundamentó en la selección de la muestra; en este caso la población objeto de estudio son los estudiantes de primer semestre y de terminación académica (décimo y undécimo semestre) del programa de Licenciatura en Matemáticas y Estadística de la Facultad Seccional Duitama de la UPTC. Como el objetivo del proyecto es identificar las concepciones de dichos estudiantes, la muestra fue seleccionada por conveniencia, ya que si se hubiera realizado un muestreo aleatorio en estos semestres se hubiesen seleccionado casi en su totalidad los estudiantes de dichos semestres, es así como fueron seleccionados 24 estudiantes de primer semestre y 18 de terminación académica. Aclarando que 8 estudiantes de primer semestre no reportaron asistencia en el transcurso del semestre.

\section{Cuestionario}

Para el diseño del cuestionario, se tuvieron en cuenta las categorías mencionadas en el marco teórico como ejes temáticos para cada una de las preguntas, las cuales estaban formadas por cuatro 


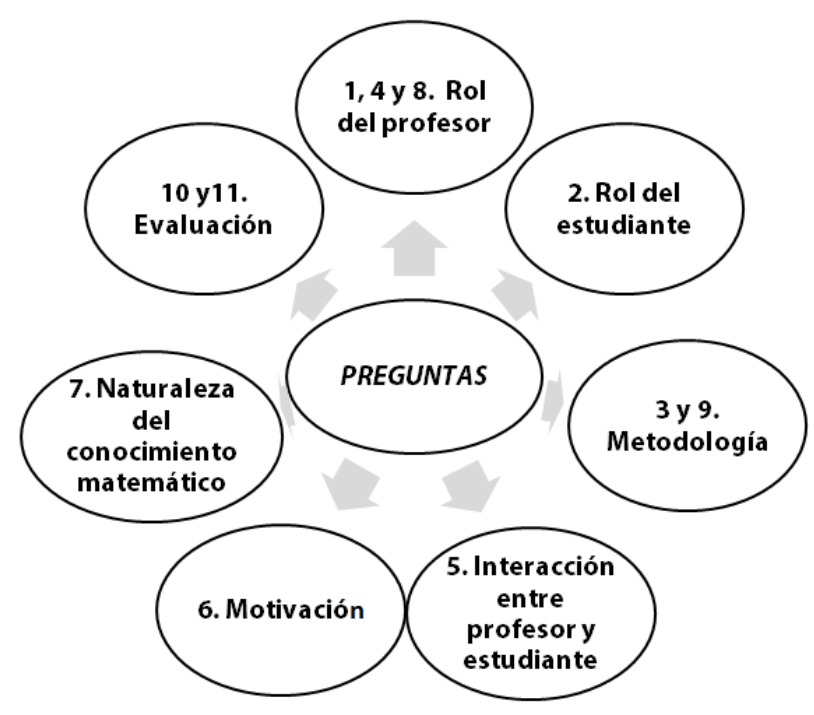

Figura 1. Eje temático de las preguntas

ítems: dos pertenecientes a la visión estática y dos a la visión dinámica, y un objetivo desde la parte didáctica, como se observa en la figura 1.

Con el propósito de evitar sesgo a la hora de responder el cuestionario, en su elaboración se tuvieron en cuenta algunas definiciones que se manejan en las asignaturas del programa, las cuales se incluyeron en el mismo ya que los estudiantes que se encuentran en primer semestre aún no las saben; estas definiciones son:

Axioma: Principio básico que es asumido como verdadero sin recurrir a demostración alguna.

Teorema: Proposición demostrable partiendo de axiomas o de otros teoremas ya demostrados.

En la elaboración del cuestionario se usó de lenguaje claro y preciso, no ambiguo, de tal forma que los estudiantes no trataran de diferenciar las subcategorías o dar la posibilidad de responder desde el deber ser más no en lo que se hace.

De otra parte, se emplea la escala Likert de 1 a 9 donde 1 es totalmente en desacuerdo y 9 es totalmente de acuerdo. Se decide utilizar esta escala ya que se encuentra dentro de la escala de medida no-métrica, y a su vez es usada para medir actitudes hacia objetos, hechos o ideas. Las ventajas que proporciona el uso de esta escala no comparativa son la facilidad en la construcción y administración, además, estas escalas son usadas en investigaciones sociológicas, humanas, sociales, psicológicas y en la educación, ya que se evalúan las actitudes y los comportamientos de las personas.

Una vez diseñado el cuestionario se realizó una prueba preliminar para detectar falencias; ésta prueba seadelantó con estudiantes de sexto semestre que cursan la asignatura de ecuaciones diferenciales. Como resultado se decidió reestructurar la pregunta inicial, quedando así: ¿Cuántos años trascurrieron entre la finalización de su bachillerato y su ingreso a la carrera?. Además se decidió agregar una última pregunta, relacionada con los aspectos a evaluar.

El cuestionario definitivo mostrado en la tabla 1, se aplicó a 42 estudiantes, en una sesión de clase, en las asignaturas de Proyecto Pedagógico Investigativo I, Vly VII. 


\begin{tabular}{|c|c|}
\hline PREGUNTA & ÍTEM \\
\hline $\begin{array}{l}\text { P1. Según su opinión, para enseñar } \\
\text { matemáticas usted, como profesor, } \\
\text { debe: }\end{array}$ & $\begin{array}{l}\text { a. Hacer énfasis en las definiciones formales y rigurosas, y la lógica } \\
\text { de los procedimientos matemáticos. } \\
\text { b. Aclarar las ideas, afirmando los conceptos, proporcionando } \\
\text { terminología y presentando la formalización requerida. } \\
\text { c.Proporcionar a los estudiantes situaciones que les permitan } \\
\text { utilizar sus conocimientos previos. } \\
\text { d.Utilizar sólo el libro de texto para guiar y orientar sus clases, } \\
\text { centrándose en preparar y transmitir la información a los } \\
\text { estudiantes. }\end{array}$ \\
\hline $\begin{array}{c}\text { P2. Según su opinión, para el } \\
\text { aprendizaje efectivo de las } \\
\text { matemáticas el estudiante debe: }\end{array}$ & $\begin{array}{l}\text { a. Reflexionar sobre su proceso de aprendizaje. } \\
\text { b. Aprender solamente lo que el profesor explica. } \\
\text { c. Realizar muchos ejercicios sobre el tema de estudio, siguiendo el } \\
\text { modelo expuesto por el profesor. } \\
\text { d.Utilizar procesos de exploración y argumentación. }\end{array}$ \\
\hline $\begin{array}{l}\text { P3. Según su opinión, las } \\
\text { actividades más adecuadas para } \\
\text { enseñar matemáticas son: }\end{array}$ & $\begin{array}{l}\text { a.La resolución de problemas y ejercicios para adquirir destrezas. } \\
\text { b.La construcción colectiva de un concepto a través de } \\
\text { situaciones problema. } \\
\text { c. El trabajo en grupo donde cada integrante tiene una función, sin } \\
\text { que entre ellos se socialice o discuta. } \\
\text { d. Las que encuentran utilidad y conexión con situaciones reales. }\end{array}$ \\
\hline $\begin{array}{l}\text { P4. Según su opinión, usted como } \\
\text { profesor considera que los } \\
\text { estudiantes necesitan estudiar } \\
\text { matemáticas porque: }\end{array}$ & $\begin{array}{l}\text { a. Son de utilidad social y profesional. } \\
\text { b. Son útiles para aquel que decida realizar una carrera de } \\
\text { "ciencias", pero no para el resto de estudiantes. } \\
\text { c. Incrementará sus posibilidades de trabajo. } \\
\text { d.El carácter formativo y riguroso de la materia, estructura el } \\
\text { pensamiento del estudiante. }\end{array}$ \\
\hline $\begin{array}{l}\text { P5. Según su opinión, como } \\
\text { profesor, la relación que establece } \\
\text { con sus estudiantes le permite: }\end{array}$ & $\begin{array}{l}\text { a.Propiciar un ambiente de confianza en donde se respetan los } \\
\text { aportes realizados por los estudiantes. } \\
\text { b.Asignar tareas para ser evaluadas. } \\
\text { c. Asumir la autoridad en las actividades que se realicen en clase. } \\
\text { d.Generar discusión y desarrollar habilidades comunicativas. }\end{array}$ \\
\hline $\begin{array}{l}\text { P6. Según su opinión, usted, } \\
\text { comoprofesor, siente satisfacción } \\
\text { del trabajo realizado cuando: }\end{array}$ & $\begin{array}{l}\text { a. Verifica que se aprende solamente lo que usted explica. } \\
\text { b. Percibe interés y participación de los estudiantes. } \\
\text { c. Observa que los estudiantes avanzan en el aprendizaje. } \\
\text { d. Los estudiantes obtienen buenos resultados en la evaluación. }\end{array}$ \\
\hline
\end{tabular}


P7. Según su opinión, las matemáticas son:

P8. Según su opinión, en la enseñanza de las matemáticas, el error se considera:
a. Un conjunto de ideas lógicas a partir de las cuales se pueden hacer demostraciones.
b. Un producto de proposiciones que se validan bajo determinadas condiciones hechas a partir de la experimentación.
c. Una construcción social, un producto cultural, susceptible al error, dependiente del contexto.
d. Un conjunto de axiomas, definiciones, teoremas y fórmulas.

a.Un medio para diagnosticar el aprendizaje, valorar y proponer nuevas estrategias.
b.Obstáculo para el aprendizaje.
c. Potencializador de los aprendizajes del estudiante.
d. Elemento para sancionar al estudiante.

P9. Según su opinión,al abordar un problema matemático, se debe:

\section{P10. Según su opinión, los} instrumentos que se deben utilizar al evaluar son:
a. Resolver sólo efectuando operaciones.
b. Interpretar y analizar el procedimiento adecuado.
c. Descubrir cuál es la operación correcta.
d. Utilizar los conocimientos matemáticos ya adquiridos.

a.Test estandarizados y pruebas generales.

b. Pruebas escritas, orales y actividades complementarias.

c. Proyectos que articulen la teoría y la práctica.

d.Resolución de problemas en el tablero.

a. Definiciones de los conceptos, enunciación de propiedades y las demostraciones.
P11. Según su opinión, en matemáticas se evalúa:

b. El uso del conocimiento matemático en la resolución de nuevos problemas.

c. Las operaciones matemáticas y la aplicación correcta de algoritmos en numerosos y variados ejercicios.

d. El desempeño según indicadores de logro y competencias matemáticas.

Tabla 1. Preguntas del cuestionario

Ya que en el cuestionario los ítems se ubicaron de manera aleatoria para efectos de que no hubiese sesgo, en el análisis de los datos, se ordenaron los ítems en una tabla clasificándolos de acuerdo a la intención de cada uno, como se observa en la tabla 2: 
b.Aprende solamente lo que el profesor explica.

2. " c.Realiza muchos ejercicios sobre el tema de estudio, siguiendo el modelo expuesto por el profesor.

a.La resolución de problemas y ejercicios para adquirir destrezas.

3. " c.El trabajo en grupo donde cada integrante tiene una función, sin que entre ellos se socialice o discuta.

b.Son útiles para el que decida realizar una carrera de "ciencias", pero no para el resto 4. " de estudiantes.

d.Incrementará sus posibilidades de trabajo.

b.Asignar tareas para ser evaluadas.

5. " c.Asumir la autoridad en las actividades que se realicen en clase.

a.Verifica que se aprende solamente lo que él explica.

6. " d.Los estudiantes obtienen buenos resultados en la evaluación.

a.Un conjunto de ideas lógicas a partir de las cuales se pueden hacer demostraciones. Logicísta

7. " d.Un conjunto de axiomas, definiciones, teoremas $y$ formulas. Formalista

b.Obstáculo para el aprendizaje.

8. " d.Elemento para sancionar al estudiante.

b.Situaciones problema que involucran la construcción colectiva de un concepto.

d.La utilidad y conexión con situaciones reales.

a.Son de utilidad social y profesional. c.El carácter formativo y riguroso de la materia estructura el pensamiento del estudiante.

a.Propiciar un ambiente de confianza en donde se respetan los aportes realizados por los estudiantes.

d.Generar discusión y desarrollar habilidades comunicativas.

b.Percibe aprecio, interés y participación de los estudiantes.

c.Observa que los estudiantes avanzan en el aprendizaje.

b.Un producto de proposiciones que se validan bajo determinadas condiciones hechas a partir de la experimentación. Empirismo.

c.Una construcción social, un producto cultural, susceptible al error, dependiente del contexto. Cuasiempirismo.

a.Un medio para diagnosticar el aprendizaje, valorar y proponer nuevas estrategias.

c.Potencializador de los aprendizajes del estudiante.

Tabla 2. Orden de los ítems 


\section{Resultados y discusión}

El análisis de los datos se realizó en dos etapas, la primera consistió en el análisis descriptivo y la segunda en la interpretación de los resultados según la implementación de las técnicas multivariadas.

\section{Análisis descriptivo}

En este análisis se halló el promedio y la desviación estándar de cada una de las preguntas en los dos grupos: grupo 1 conformado por estudiantes de primer semestre, y grupo 2 conformado por estudiantes de últimos semestres. Se evidenció que hay diferencias significativas en preguntas como la P1 (a), P2 (b), P3 (c), P7 (d), y P10 (b); Entiéndase por $\mathrm{P} 1$ (a) pregunta 1 ítem a , y así sucesivamente, de acuerdo con la nomenclatura de las tablas 1 y 2 .

\section{Análisis multivariado}

En este análisis se realiza la prueba de normalidad multivariada y, con base en dichos resultados, se efectúa el análisis de componentes principales y análisis discriminante.

A continuación aparecen los códigos y los respectivos resultados:

datos=read.table('datos.txt',header $=T$ ); datos=datos[-2]

$\mathrm{d} 1=$ datos$[1: 24, c(2: 24,29: 31,33: 38,40: 42)] ;$ mshapiro.test $(\mathrm{t}(\mathrm{d} 1))$

Shapiro-Wilk normality test

$\mathrm{W}=0.9678, \mathrm{p}$-value $=0.6136$

El resultado de esta prueba (1) manifiesta una multi-normalidad para las variables que se mencionan en el análisis descriptivo y se produce luego de una exploración exhaustiva, que está dentro del conjunto de variables en análisis para el grupo 1. En el caso del grupo 2, dada la dispersión en las posiciones de los estudiantes, la multinormalidad se logra eliminando el $37 \%$ de las variables en estudio dado que manifiestan una multi-colinealidad (2) con las demás variables objeto de estudio.

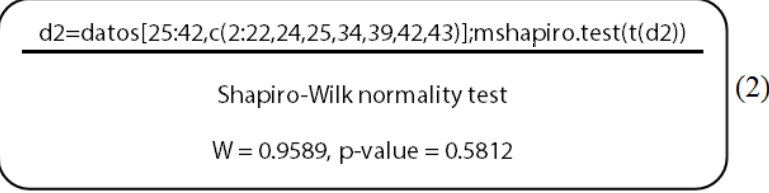

Con estos resultados a continuación se indican los análisis multivariados, a saber: el análisis de componentes principales, el análisis discriminante lineal y el análisis de correspondencias.

\section{Análisis discriminante}

Los resultados de este análisis muestran que los estudiantes del grupo 1 consideran importante la P5 (b y c), donde afirman que la relación que como profesores establecen con los estudiantes les permite asignar tareas para ser evaluadas, asumiendo la autoridad en las actividades que se realicen en clase, además en la P8 (a y d), piensan que el error es un medio para diagnosticar el aprendizaje, valorar y proponer nuevas estrategias, siendo a su vez un elemento para sancionar al estudiante.

De igual manera, en la P4 (a y c) los estudiantes piensan que el profesor considera que los estudiantes deben estudiar matemáticas ya que son de utilidad social y profesional incrementando sus posibilidades de trabajo; otro caso es la P6 (b) en donde los estudiantes consideran significativo que el profesor siente satisfacción cuando percibe el interés y participación de los estudiantes.

Mientras que en el grupo 2, los estudiantes que se encuentran en décimo semestre tienen como preguntas diferenciales las siguientes:

En la P2 (a y b), los estudiantes consideran que para aprender de manera efectiva las matemáticas se debe reflexionar sobre su proceso de aprendizaje limitado a su vez por la concepción de que se debe aprender solamente lo que el profesor explica, 
como se puede observar en el formato del cuestionario final. De igual manera, en la P1 (d), los estudiantes piensan que el profesor no debe ser tan sólo un ente transmisor del conocimiento, además la P7 (d) el grupo 2 se caracteriza en especial porque los estudiantes consideran que las matemáticas no son solamente un conjunto de axiomas, definiciones, teoremas y fórmulas, aislados de un entorno real; además manifiestan que en las matemáticas no se deben evaluar las definiciones de los conceptos, enunciación de propiedades y demostraciones. La P5 (a), aunque es diferenciadora, está orientada en sentido negativo puesto que los estudiantes consideran que no es conveniente sostener un ambiente de confianza en medio del respeto por los aportes de los estudiantes.

Entre tanto que en el grupo 2 se encuentran las siguientes preguntas diferenciales:

En la P3 (a, b, c y d), los estudiantes piensan que las actividades más adecuadas para enseñar las matemáticas son la resolución de problemas y ejercicios para adquirir destrezas, la construcción colectiva de un concepto a través de situaciones problema, teniendo en cuenta el trabajo en grupo donde cada integrante tiene una función, sin que entre ellos se socialice o discuta, y las que encuentran utilidad y conexión con situaciones reales; de igual manera en la P10 (d) ellos consideran que los instrumentos que se deben utilizar al evaluar es la resolución de problemas en el tablero. En la P8 (b), consideran que la enseñanza de las matemáticas el error es considerado como un obstáculo para el aprendizaje.

\section{Análisis de componentes principales}

En este análisis se observa que dados los dos primeros componentes la P3 (b, d) y la P7 (d) tiene un comportamiento bastante disímil, y dentro del primer grupo es interesante revisar la posición de la observación 12 a fin de determinar su percepción de manera particular. De acuerdo con estos dos componentes las preguntas que mayor importancia tienen son P2 (b), P4 (a y b), lo que significa que en el aprendizaje significativo de las matemáticas el estudiante debe aprender solamente lo que el profesor explica, el estudiante debe estudiar las matemáticas porque son de utilidad social y profesional y son útiles para aquel que decida realizar una carrera de ciencias, pero no para el resto de los estudiantes.

En el grupo 2 se observa que las concepciones de los estudiantes son muy heterogéneas y además, se observa que las preguntas tienen un comportamiento no aglomerado, es decir que en los estudiantes no hay calificaciones similares, lo que implica que con estas dos componentes principales se explique una muy pequeña parte de la variabilidad dando relevancia especialmente a valores muy grandes para P2 (a y b), P6 (a), P9 (a), P3 (b) y P4 (a), que inciden de manera negativa en la puntuación por componente, lo que significa que los estudiantes piensan que para el aprendizaje de las matemáticas el estudiante no debe reflexionar sobre su proceso de aprendizaje y aprender solamente lo que el profesor explica, teniendo en cuenta que las actividades más adecuadas para enseñar matemáticas no son la construcción colectiva de un concepto a través de situaciones problema. Se evidenció también que como profesores no sienten satisfacción del trabajo realizado cuando perciben interés y participación por parte de los estudiantes, ellos piensan que al abordar un problema matemático no se debe resolver solo efectuando operaciones.

La pregunta P3 (a y b), P2 (b), y la pregunta P4 (a) tiene un comportamiento bastante disímil, y dentro del grupo completo es interesante revisar la posición de la observación 14, 40 y 36 a fin de determinar su percepción de manera particular, las pregunta P3 (b) de manera positiva en la calificación del componente, lo que significa que las actividades más adecuadas para enseñar matemáticas son la construcción colectiva de un concepto a través de situaciones problema. 


\section{Análisis de correspondencias múltiples}

En este análisis no se pueden establecer claras diferencias entre los grupos, con respecto a las concepciones sobre la naturaleza de las matemáticas y su didáctica, lo que quiere decir que hay semejanza en la calificación de algunas de las variables consideradas, siendo más dispersas las calificaciones del primer grupo.

Con el análisis multivariado se evidencia la diferencia en la manera de pensar de los estudiantes que ingresan a primer semestre y los estudiantes que se encuentran en terminación académica, lo cual muestra la forma en que el programa ha influido de manera positiva en el cambio de las concepciones y creencias de los estudiantes en el transcurso del mismo, teniendo en cuenta la importancia que debe tener la didáctica de las matemáticas en el aula de clase como futuros docentes.

Se resalta la importancia del análisis multivariado en esta investigación, ya que permitió establecer las tendencias en las creencias y concepciones de los estudiantes en cada uno de los grupos considerados. En este caso, los análisis discriminante y el de componentes principales permitieron identificar con precisión las diferencias entre los dos grupos, para establecer las preguntas que tenían más significado; mientras que con el análisis de correspondencias múltiples solo se establecieron conclusiones a nivel general y no permitió identificar diferencias marcadas para los grupos estudiados. De otro lado, el software que se usó fue el adecuado ya que posee muchas herramientas que permitieron el análisis de los datos.

\section{Conclusiones}

En el desarrollo del proyecto, a través del análisis multivariado, se evidenciaron claras diferencias en la manera de pensar de los estudiantes en los diferentes grupos, con respecto a las concepciones sobre la naturaleza de las matemáticas y su didáctica. En los estudiantes que comienzan la carrera se observa que tienden a tener una visión estática, del cómo enseñan es decir, consideran que esta está basada exclusivamente en la transmisión de los conocimientos, considerando como básica la comunicación en un solo sentido (profesorestudiante) y con respecto a la evaluación, consideran que es un proceso que consiste en comprobar por parte del profesor que el estudiante es capaz de repetir sus explicaciones, teniendo en cuenta como instrumentos las pruebas escritas. Se evidenció que las preguntas que mayor importancia tienen son P2 (b) en donde se hace referencia a que el estudiante aprende solamente lo que el profesor explica y la P4 (a y b) en donde el profesor considera que las matemáticas son de utilidad social y a su vez son útiles para el que decida realizar una carrera de "ciencias", pero no para el resto de estudiantes.

Por el contrario, los estudiantes de últimos semestres de la carrera manifiestan una visión dinámica del cómo se enseña la matemática, vista como un proceso de construcción de conocimientos, en donde intervienen: la exploración, la resolución, la discusión de las tareas y el desarrollo de investigaciones en el aula. Pero consideran que el aprendizaje es esencialmente un proceso activo para construir compensaciones y estrategias, como se evidencia especialmente en las preguntas P2 ( $a$ y b) en donde el estudiante reflexiona sobre su proceso de aprendizaje y aprende solamente lo que el profesor explica, en P1 (d) se considera que el profesor debe utilizar solo el libro de texto para guiar y orientar sus clases, centrándose en preparar y transmitir la información a los estudiantes, y P7 (d) en donde se considera que la naturaleza de las matemáticas son un conjunto de axiomas, definiciones, teoremas y fórmulas, vista desde una corriente formalista.

Dados los resultados del análisis discriminante se evidencia la diferencia en la manera de pensar de los estudiantes que ingresan a primer semestre y 
los estudiantes que se encuentran en terminación académica, lo cual muestra el impacto positivo del programa de Licenciatura en Matemáticas y Estadística, en cuanto al cambio de las concepciones y creencias de los estudiantes en el transcurso del mismo, resaltando la importancia que debe tener la didáctica de las matemáticas en el aula de clase como futuros docentes.

Con esta investigación también se resalta la importancia de continuar adelantando estudios sobre las concepciones de los profesores en formación en el área de las matemáticas, ya que con ellas será posible alcanzar el objetivo de transformar la visión de los estudiantes de secundaria con respecto al aprendizaje de las mismas.

\section{Agradecimientos}

Los resultados aquí reportados corresponden al proyecto SGI 817, inscrito en la Dirección de Investigaciones de la UPTC.

\section{Referencias}

Azcarate, P., \& Cardeñoso, J. (1998). Formación inicial de profesores de matemáticas, finalidades, limitaciones y obstáculos. Investigación en la escuela, 35, 75-85.

Azcárate, C., Garcia, L., \& Moreno, M. (2006). Creencias y concepciones del conocimiento profesional de profesores que enseñan cálculo diferencial a estudiantes de ciencias económicas. Revista Latinoamericana de Investigación Educativa, 9(1), 85-116.

Bautista, L. (1998). Diseños de muestreo estadístico. Bogotá D. C.: Ciudad universitaria.

Blanco, L. (2002). Educación Matemática y formación inicial del profesorado de primaria, secundaria y bachillerato. Revista Interuniversitaria de Formación de Profesorado, (43), 173-179.
Caballero, A., Blanco, L. J. y Guerrero, E. (2007), Las actitudes y emociones ante las Matemáticas de los estudiantes para Maestros de la Facultad de Educación de la Universidad de Extremadura. Comunicación presentada en el Grupo de Trabajo "Conocimiento y desarrollo profesional del profesor", en el XI SEIEM. Simposio de Investigación y Educación Matemática, celebrado en la Universidad de La Laguna los días 4 al 7 de Septiembre de 2007.

Carrillo, J., \& Contreras, L. (1995). Un modelo de categorías e indicadores para el análisis de las concepciones del profesor sobre la matemática y su enseñanza. Revista Educación Matemática, 7(3), 79-92.

Cerón, D., Mesa Y., \& Rojas C. (2012). La naturaleza del conocimiento matemático y su impacto en las concepciones del profesor. Rev.investig. desarro.innov, 2(2), 49-59.

D’Amore, B., \& Fandiño, M. (2004). Cambio de convicciones en futuros profesores de matemáticas de la Escuela Secundaria Superior. Revista Epsilon, (58), 25-43.

Díaz, G. (2002). Estadística Multivariada: inferencia y métodos. Bogotá: Panamericana Formas e Impresos S.A.

Dodera, G., Burroni, A., Lázaro, M., \& Piacentini, A. (2008). Concepciones y creencias de profesores sobre la enseñanza y aprendizaje de la matemática. Revista Premisa de la sociedad Argentina de educación matemática, (39). Recuperado de: http://www.soarem.org.ar/ Documentos/39\%20Dodera.pdf

Flores, P. (1998). Creencias y concepciones de los futuros profesores sobre las matemáticas, su enseñanza y aprendizaje. Evolución durante las prácticas de enseñanza. Departamento de Didáctica de la Matemática. Universidad de Granada. 
Gámez, P., Moreno, M., \& Gil, F. (2003). Concepciones de los futuros profesores sobre la enseñanza y aprendizaje de las matemáticas. Investigación en educación matemática: Séptimo simposio de la sociedad española de investigación en educación matemática. ISBN 84-338-3019-8, p. 213-226.

Gil, F., \& Rico, L. (2003). Concepciones y creencias del profesorado de secundaria sobre enseñanza y aprendizaje de las matemáticas. Enseñanza de las ciencias: Revista de investigación y experiencias didácticas, 21(1), 27-47. Recuperado de: http:// www.raco.cat/index.php/ensenanza/article/view File/21885/21719.

Godino, J., Batanero, C. \& Font, V. (2004). Fundamentos de la enseñanza y el aprendizaje de las matemáticas. Recuperado de: http://www. matesup.utalca.cl/modelos/articulos/fundament os.pdf

Jiménez, A. (septiembre, 2009). Las concepciones sobre la naturaleza de la matemática y su influencia en el salón de clase. Memorias VII Encuentro Nacional de Educación Matemática y
Estadística. Universidad Pedagógica y Tecnológica DeColombia.Tunja.

Llinares, S. (1991). La formación de profesores de matemáticas. Sevilla: GID-Universidad de Sevilla. Marcelo, C. El pensamiento del profesor. Barcelona. Ceac.

Moreno, M., \& Azcarate, C., (2003). Concepciones y creencias de los profesores universitarios de matemáticas acerca de la enseñanza de las ecuaciones diferenciales. Enseñanza de las ciencias, 21(2), 265-280.

National Council Of Teachers Of Mathematics, (NTCM). (1991)

Salvador, M., Bigné, E., Levy, J-P., Cuenca, A. C., \& Miguel, M. J. (1997). Investigación de mercados. España: McGraw-Hill, Interamericana de España S.A.

Zapata, M., Blanco, L., \& Contreras, L. (2008). Los estudiantes para profesores y sus concepciones sobre las matemáticas y su enseñanzaaprendizaje. REIFOP, 12(4), 109-122. 